\title{
MICROFLUIDIC DELIVERY OF NANOMEDICINE: THE LITTLE DROP OF FLUID THAT COULD
}

\author{
Abraham P. Lee ${ }^{1,2}$, Albert T.-H. Hsieh ${ }^{l}$, Armando Tovar ${ }^{l}$, and Kanaka Hettiarachchi ${ }^{1}$ \\ ${ }^{1}$ Department of Biomedical Engineering, \\ ${ }^{2}$ Department of Mechanical \& Aerospace Engineering \\ DARPA/Industry Micro/nano Fluidics Fundamentals Focus (MF3) Center \\ University of California at Irvine, 3120 Natural Sciences II, Irvine, CA 92697-2715, U.S.A.
}

\begin{abstract}
Medicine as we know it is a series of standard procedures (check-ups, sample collection, lab tests, drugs, and treatment) that are generally stand alone with little to no connectivity between the different "stations". However, these medical stations are starting to converge in tiny fluid drops delivered by microfluidic chips. Over the years, lab-on-a-chip devices have demonstrated strong potential for rapid detection and point-ofcare diagnostics. These devices are capable of detection and manipulation at the cellular and molecular level with high throughput. More recently, digital "droplet" microfluidic chips have shown promise to "nanomanufacture" the "magic bullet", hybrid micro/nano particles that incorporate imaging, detection, diagnostics, and targeted delivery of drugs for advanced therapies. I will introduce three active microfluidic "nanomedicine" platforms in my lab: DNA analysis and detection, protein serodiagnostics to screen antibodies, and a acoustically active lipospheres for ultrasound imaging and drug delivery.
\end{abstract}

\section{DNA HYBRIDIZATION DETECTION}

The monodispersed picoliter microfluidic droplet generation system developed by our group and others [0] can serve as a promising micro-reactor for biological and chemical assays. It employs pressure-driven flow to inject aqueous solutions into aqueous immiscible solutions and form picoliter microdroplets. The biological or chemical reagents are all encapsulated in microdroplets and each droplet is isolated by the immiscible liquid (e.g. mineral oil), thus greatly reducing sample contamination on the microchannel side walls and eliminating reagent dispersion problems. The system is useful not only for DNA sample identification, but also for quantitative analysis. The liquid-liquid reaction rate of DNA hybridization in homogeneous liquid is about 40-fold faster than the hybridization rate in a solid-liquid interface [0]. Hence, it is advantageous to improve the DNA sample/sensing probe hybridization rate using this technique rather than the conventional immobilized-probe approach. Compared to conventional microfluidics with continuous laminar flows, the microdroplet allows rapid mixing among reagents in droplet [0] . The microdroplet formation system that we developed is capable of generating over five hundred monodisperse droplets per second with a size deviation of less than two percent [0]. However, because of the fundamental differences between the microdroplet generation system and other conventional techniques, a liquid-liquid reaction based DNA sensing probe is needed to conduct DNA detection in microdroplets.

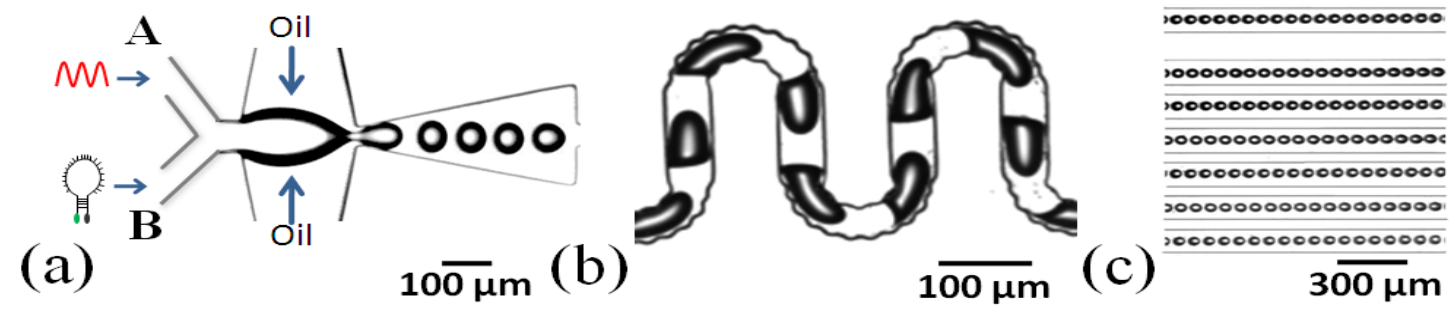

Fig. 1 The merged $A$ and B streams are broken off by side streams and form microdroplets. (a) Consecutive micrographs of droplet generation. The generation time is $2.66 \mathrm{~ms} /$ droplet. (b) DNA and MB are rapidly mixed in sawtooth edged U-shaped channel (c) Picoliter droplets moving in hybridization region [0].

In order to achieve rapid DNA detection in homogeneous liquid phase with single nucleotide mismatch sensitivities, we used molecular beacon (MB) as the DNA sensing probe in the microdroplets [0]. The target DNA and $\mathrm{MB}$ were all encapsulated in monodispersed picoliter droplet emulsions to reduce sample volume and to enhance the DNA detection efficiency. In our paper [0], we demonstrate a fast DNA sample and mutant detection in a few seconds, and evaluate dynamic MB-DNA duplex formation using Label-Free DNA Analysis in Microdroplet (LFDAM) system. The more stable the MB-DNA duplex, the stronger the MB fluorescence restored. The measured fluorescence intensity is directly proportional to sample concentration, droplet volume, droplet generation rate, and fluorescence acquisition time. Figure 1 illustrates (a) the microfluidic droplet generation device, (b) the mixing serpentine channel, and (c) a snapshot of the droplets travelling in the microchannels. Figure 2 illustrates the gradual increase in fluorescent intensity as the droplets travel in the microchannels and more time is lapsed so the MB-DNA duplex has a chance to reach equilibrium. 
(a)

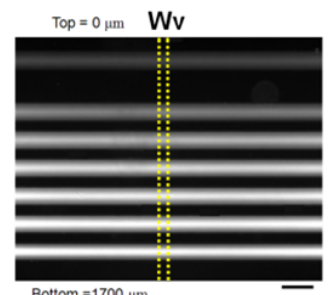

Fig. 2 The dynamic binding reaction of target DNA with $M B$ can be observed from the fluorescence change of $M B$ inside droplet. (a) The horizontal averagemultiple-line-scan method is used to measure the fluorescence intensity. The yellow dash line represents the fluorescence analysis from the top to the bottom of an image. (b) The measured result shows that the $M B$ fluorescence gradually increases downstream in the LFDAM [0].

\section{ANTIBODY SERODIAGNOSTICS ASSAYS}

A novel microfluidic actuator is designed around the use of trapped air bubbles in lateral cavities and the resultant acoustic streaming generated from an outside acoustic energy source $[0,0]$. This actuator is simple to fabricate, integrate, and operate in a compact portable configuration. The orientation of the lateral cavities to the main microchannel is used to control the bulk fluid motion within the device and the first order flow generated by the oscillating bubble is used to develop a pumping platform that is capable of driving fluid within a chip. The device is fabricated using traditional soft lithography. [0] One of the main advantages of using the LCAT platform is the simple fabrication needed for integration into existing chips. The LCATs are designed within the same layer as the main microchannel and only the single lithography step needed for the main microchannels is required to fabricate both channel features and the LCATs. The device is operated by by placing it on top of an external acoustic energy source. The operation of the device can be seen in Fig. 3 where a PZT is coated with ultrasound gel to enhance acoustic energy transfer through the bottom substrate of the chip.

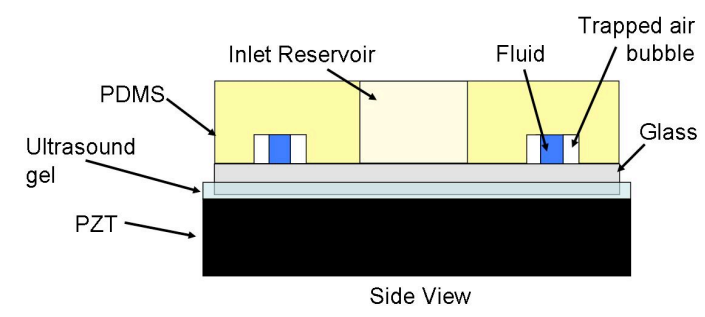

Fig. 3 Schematic of Acoustic Bubble-Array Micromixer

The demonstration of the pumping capabilities of the LCAT platform is done using cavities oriented at $15^{\circ}$ to the main channel. The orientation of the cavities utilizes the first order oscillatory flow from the air/liquid interface to produce a net flow away from the cavity interface. The design can be seen in Fig.4, where the trapped air bubble can be seen within the lateral cavity and the two cavities are oriented at a shallow angle $\left(15^{\circ}\right)$ to the main microchannel.

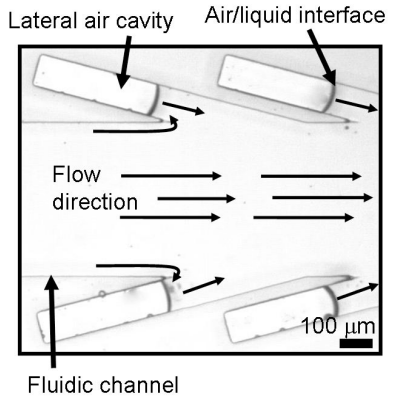

Fig. 4 LCAT pumping design with cavities at $15^{\circ}$ angles.

This pump is integrated into a recirculation immunoassay device for enhanced antibody-antigen binding through fluid flow for convection-limited transport. The device is designed to continuously deliver the analyte over a binding region. The recirculation assay device has the advantages of allowing high flow rates for increased binding efficiency while maintaining a low fixed volume. In traditional microfluidic flow through devices, the higher flow rates lead to larger volume consumption during operation. The operation of the device is performed using a protein spotted nitrocellulose pad that is bound with gold-conjugated secondary antibodies for optical confirmation of binding.

To determine the effectiveness of a recirculation assay platform (Fig. 6a) [0], it is compared to a traditional assay where gold conjugated antibodies (goat anti-human IgG (H\&L)) are delivered over a protein spotted nitrocellulose pad for 15 minutes. The comparison shown in Fig. 6B shows an increase in spot intensity using the microfluidic recirculation platform compared to the traditional benchtop assay where $50 \mu \mathrm{L}$ is incubated within a well plate for the same amount of time. The simulation and experimental results showed good agreement for antigen site binding.
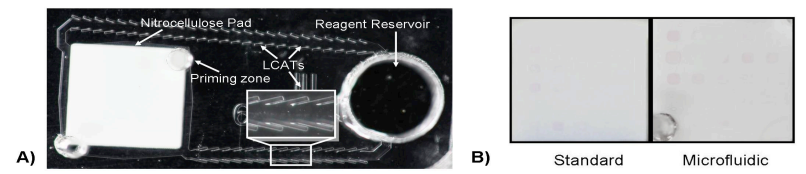

Fig. 5 (a) Image of colorimetric immunoassay chip. (b) Comparison of standard and microfluidic 1 OD gold-conjugated secondary antibody delivery.

\section{MULTILAYER MICROPARTICLES FOR NANOMEDICINE (MMN)}

The utilization of acoustically active lipospheres (AALs) (Fig. 6a) capable of delivering bioactive substances at high concentrations in the lipid-oil complex has been reported $[0,0]$. The oil layer is capable of carrying highly hydrophobic drugs such as the chemotherapeutic drug Paclitaxel, which is currently delivered to cancer patients intravenously. Paclitaxel can be dissolved at high concentrations $(70 \mathrm{mg} / \mathrm{ml})$ in the lipid-oil complex of the AALs [0]. Such vehicles can provide an alternative method of delivery of therapeutic drugs that have poor oral bioavailability.

The commonly used production technique of mechanical shaking creates a highly polydisperse microbubble population, with varying amounts of oil within many of the microbubble vehicles. Thus, the drug dosages within each vehicle are also 
not consistent. Microfluidic chambers provide an ideal platform for the controlled production of monodisperse double emulsions $[0]$ and microbubble contrast agents [0] with a much smaller size distribution. Our device (Fig. 6b) combines two hydrodynamic flow-focusing regions together and features expanding nozzle geometry to generate gas-filled dual-layer lipospheres.
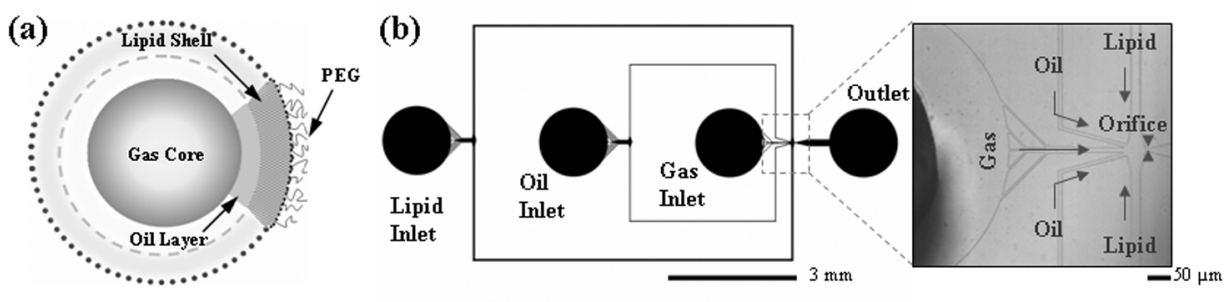

Fig. 6 (a) Schematic of an AAL and (b) PDMS-based microfluidic flow-focusing device

Microfluidic generated lipospheres are visible in brightfield (Fig. $7 b$ ), and the inner blue layer confirms the existence of the additional triacetin oil layer (Fig. 7c). The outer
DSPC lipid shell stabilizes the vehicle and the inner triacetin oil layer can contain dissolved therapeutics. The gas interior makes the vehicle acoustically active to ultrasound pulses.
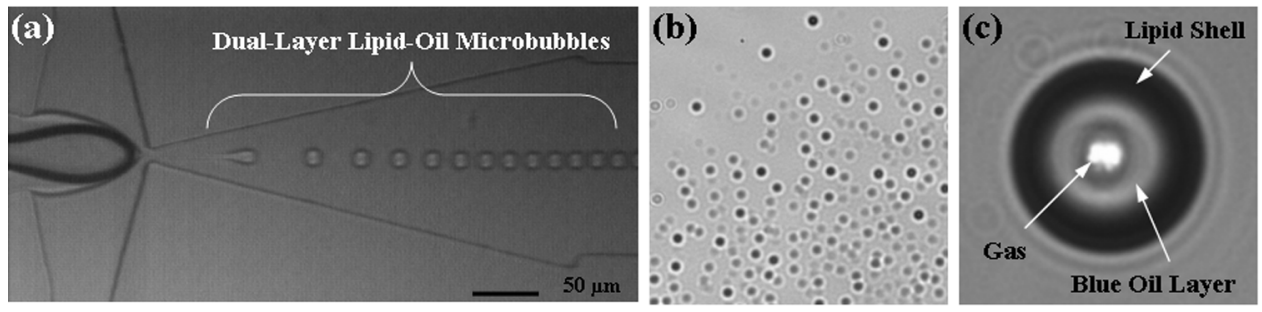

Fig. 7 (a) Production by flow-focusing and (b) generated AALs. (c) Close-up of AAL

Microbubble size and production rate are highly prone to downstream pressure conditions. The large viscosity difference between the oil and gas makes the production of dual-layer lipospheres particularly challenging. Channel geometry in addition to the liquid and gas flow rates are the main factors used to control the liposphere sizes.

Paclitaxel is a poorly water soluble drug and its large size prevents it from being accommodated within the phospholipid bilayers in liposomes. In a dual-layer vehicle, a high volume fraction of the oil phase can be used and paclitaxel exhibits good solubility in triacetin. The production method will enable more precise dosages of drug to be delivered. Conjugating a cyclicRGD peptide to the shell due to over-expression of $\alpha v \beta 3$ integrin in the tumor blood vessel endothelium is a possibility for targeted delivery.

The issue of creating long-lasting dual-layer microbubble lipospheres for drug delivery in microfluidic systems poses interesting studies concerning the impact of device geometry and scale, flow parameters, and the synergy between shell, internal components, and surrounding medium on the stabilization.

With microfluidics technology, we can produce monodisperse dual-layer microbubbles in a suitable size range for oral drug delivery or direct injection into the circulation (10 $\mu \mathrm{m}$ or less). The composition makes them ultrasonically active and able to carry a large and precise drug payload to the target site. On-going and immediate experiments are to demonstrate drug loading in the manufactured dual-layer vehicles, in vitro targeted delivery to cancer cells, in vivo biodistribution animal studies, and to demonstrate that the drug Paclitaxel can remain, or be slowly released over time.

\section{CONCLUSIONS}

Microfluidic droplet interfaces can deliver a broad range of nanomedicine applications, including the detection of biomolecules (e.g. DNA), the diagnostics of antibodies specific to infectious diseases, and the targeted therapy for cancer and other diseases with molecular markers. The microfluidic chips generate monodisperse fluid volumes and controlled fluid motion that form integration modules for future healthcare solutions.

\section{REFERENCES}

[1] Shia-Yen Teh, Robert Lin, Lung-Hsin Hung, and Abraham P. Lee, "Droplet Microfluidics", Lab on a Chip, vol. 8 (2), pp. 198-220, 2008.

[2] Gao, Y., L. K. Wolf, et al. (2006). "Secondary structure effects on DNA hybridization kinetics: a solution versus surface comparison." Nucleic Acids Res. 34(11): 33703377.

[3] Tice, J. D., H. Song, et al. (2003). "Formation of droplets and mixing in multiphase microfluidics at low values of the Reynolds and the capillary numbers." Langmuir 19(22): 9127-9133.

[4] Tan, Y. C., J. S. Fisher, et al. (2004). "Design of microfluidic channel geometries for the control of droplet volume, chemical concentration, and sorting." Lab Chip 4(4): 292-298.

[5] Abraham P. Lee, "Microfluidic Cellular and Molecular Detection for Lab-on-a-Chip Applications", the 31st Annual International Conference of the IEEE Engineering in Medicine and Biology Society, Minneapolis, Minnesota, USA, 2-6 September, 2009. 
[6] Albert T. Hsieh, Patrick J. Pan, Abraham P. Lee, "Rapid label-free DNA analysis in picoliter microfluidic droplets using FRET probes", Microfluidics and Nanofluidics, vol. 6 (3), pp. 391-401, 2009.

[7] Armando R. Tovar, Algimantas Jasinskas, Philip L. Felgner, and Abraham P. Lee "Microfluidic 5-Step Colorimetric Immunoassay with On Chip Pumping", The Thirteenth International Conference on Miniaturized Systems for Chemistry and Life Sciences (uTAS 2009) ICC Jeju, Jeju, Korea, November 1-5, 2009

[8] Armando Tovar, Maulik Patel, and Abraham P. Lee, "Acoustic cavity transducers for the manipulation of cells and biomolecules",

[9] Whitesides, G. M. et. al., « Soft lithography in biology and biochemistry," Annual Review of Biomedical Engineering, 3, 335-373, 2001.

[10] Yuen, P.K. et al, "Microfluidic Devices for Fluidic Circulation and Mixing Improve Hybridization Signal Intensity on DNA Arrays," Lab on a Chip, 3, 46-50, 2003.

[11] Kanaka Hettiarachchi, Esra Talu, Marjorie L. Longo, Paul A. Dayton, Abraham P. Lee, "Manufacture of Dual-Layer Microbubble Lipospheres as Drug Delivery Vehicles in Microfluidic Devices", The Eleventh International Conference on Miniaturized Systems for Chemistry and Life Sciences (uTAS 2007), p.191-193, Paris, France, October 7-11, 2007
[12] Evan C. Unger, Thomas P. McCreery, Robert H. Sweitzer, Veronica E. Caldwell, and Yunqiu Wu, "Acoustically Active Lipospheres Containing Paclitaxel: A New Therapeutic Ultrasound Contrast Agent," Investigative Radiology, Vol. 33, pp. 886-892 (1998).

[13] M. J. Shortencarier, P. A. Dayton, S. H. Bloch, P. A. Schumann, T. O. Matsunaga, and K. W. Ferrara, "A method for radiation-force localized drug delivery using gas-filled lipospheres," IEEE Trans Ultrason Ferroelectr Freq Control, Vol. 51, pp. 822-831 (2004).

[14] T. Nisisako, S. Okushima and T. Torii, "Controlled formulation of monodisperse double emulsions in a multiple-phase microfluidic system," Soft Matter, Vol. 1, pp. 23-27 (2005).

[15] K. Hettiarachchi, E. Talu, M. L. Longo, P. A. Dayton, and A. P. Lee, "On-chip generation of microbubbles as a practical technology for manufacturing contrast agents for ultrasonic imaging," Lab Chip, Vol. 7, pp. 463-468 (2007). 